\title{
A multiscale 3D chemotaxis assay reveals bacterial navigation mechanisms
}

\section{Marianne Grognot}

Rowland Institute at Harvard https://orcid.org/0000-0002-7491-1224

Katja Taute ( $\nabla$ taute@rowland.harvard.edu )

Harvard University https://orcid.org/0000-0003-2230-717X

\section{Article}

Keywords: Bacteria Motility, 3D Trajectories, Surface Effects

Posted Date: September 11th, 2020

DOI: https://doi.org/10.21203/rs.3.rs-61942/v1

License: (c) (i) This work is licensed under a Creative Commons Attribution 4.0 International License. Read Full License

Version of Record: A version of this preprint was published at Communications Biology on June 3rd, 2021. See the published version at https://doi.org/10.1038/s42003-021-02190-2. 


\title{
A multiscale 3D chemotaxis assay reveals bacterial navigation mechanisms
}

\author{
Marianne Grognot \& Katja M. Taute* \\ Rowland Institute at Harvard University, 100 Edwin H. Land Blvd, Cambridge, MA 02142, US \\ *corresponding author: taute@rowland.harvard.edu
}

\begin{abstract}
How motile bacteria navigate environmental chemical gradients has implications ranging from health to climate science, but the underlying behavioral mechanisms are unknown for most species. The well-studied navigation strategy of Escherichia coli forms a powerful paradigm that is widely assumed to translate to other bacterial species. This assumption is rarely tested because of a lack of techniques capable of bridging scales from individual navigation behavior to the resulting population-level chemotactic performance.

Here, we present such a multiscale 3D chemotaxis assay by combining high-throughput 3D bacterial tracking with microfluidically created chemical gradients. Large datasets of 3D trajectories yield the statistical power required to assess chemotactic performance at the population level, while simultaneously resolving the underlying 3D navigation behavior for every individual. We demonstrate that surface effects confound typical 2D chemotaxis assays, and reveal that, contrary to previous reports, Caulobacter crescentus breaks with the E. coli paradigm.
\end{abstract}

\section{Introduction}

Chemotaxis enables bacteria to navigate external chemical fields and is now recognized as a key factor driving interactions of bacteria with their environment and each other, with wide-ranging effects that include promoting pathogenicity ${ }^{1}$, establishing symbioses ${ }^{2}$, and shaping geochemical fluxes $^{3}$. Because individual bacterial motility behavior has a large random component, chemotaxis is usually assessed using population-level assays that average over the behavior of thousands of individuals, ranging from Adler's classic capillary assay ${ }^{4}$ to modern approaches based on video microscopy $y^{5}$. While these approaches are highly sensitive and precise in detecting small chemotactic effects, they are blind to the underlying behavioral mechanisms. Berg's pioneering 3D tracker capable of following a single bacterium swimming in 3D demonstrated the importance of resolving individual 3D motility behavior for revealing chemotactic mechanisms ${ }^{6}$. The resulting understanding that Escherichia coli chemotaxis is controlled via the bias in the rotation direction of the flagellum ${ }^{7}$ has become a dominant paradigm in bacterial chemotaxis research ${ }^{8}$. Recent findings, however, suggest that many other species, including the majority of marine bacteria, may use a different strategy ${ }^{9,10}$, highlighting the need for efficient and broadly applicable methods of characterizing chemotactic behavior.

The pervasive inter-individual variability present even in genetically identical populations ${ }^{11}$ renders throughput and sampling crucial bottlenecks in characterizing chemotactic behavior based on trajectory data. Methods aimed at revealing potentially diverse chemotactic 
mechanisms must bridge the scales between individuals and populations by capturing motility behavior of individual bacteria with the statistical power to simultaneously reveal chemotactic performance, which is typically only accessible via ensemble averages. Here we introduce a novel chemotaxis assay that enables such a multiscale approach by harnessing a recently developed high-throughput 3D tracking method ${ }^{12}$ to capture individual navigation behavior in the presence of microfluidically created chemical gradients for thousands of bacteria within minutes. After validating our approach using the well-characterized $E$. coli model system, we demonstrate its power by revealing that the chemotactic mechanism of the freshwater bacterium Caulobacter crescentus breaks with the E. coli paradigm.

\section{Results and Discussion}

\section{Multiscale assay accurately quantifies $E$. coli chemotaxis}

In our assay, typically 50-100 bacteria are tracked simultaneously in 3D in the center of a quasistatic, linear gradient field created in a microfluidic device (Fig. 1a, Supplementary Fig. 1a-d, Methods). Typically, thousands of trajectories are gathered in minutes, enabling a precise determination of the chemotactic drift velocity, $v_{d}$, as the population-averaged velocity along the direction of the gradient ( $x$ ) (Fig. 1b). We demonstrate the technique by assessing chemotaxis of the well characterized $E$. coli strain AW405 towards the non-metabolizable chemoattractant $\alpha$ methyl-DL-aspartate (MeAsp, Fig. 1b-e, Supplementary Fig. 2e). The population-averaged drift velocity of $2.7 \pm 0.3 \mu \mathrm{m} / \mathrm{s}$ (mean $\pm \mathrm{SD}$ ) for bulk trajectories in a linear gradient of $10 \mu \mathrm{M} / \mathrm{mm}$ MeAsp aligns well with values imputed from previous work (Supplementary Discussion), confirming that our technique provides an accurate quantification of chemotactic performance.

\section{E. coli's chemotactic drift decreases near the chamber surface}

Resolving the drift velocity, $v_{d}$, as a function of vertical position, $z$, reveals that the drift velocity is roughly constant in the bulk liquid, but decreases sharply near the surfaces of the sample chamber (Fig. 1d, Supplementary Fig. 2f). We attribute this decrease to trajectory curvature at the surfaces (Fig. 1c) randomizing bacterial orientations and thus leading to a decreased chemotactic response. Such curvature results from hydrodynamic interactions with the surface ${ }^{13}$ and is thus present in common 2D motility assays that increase trajectory durations by either constraining the bacteria to a thin sample chamber ${ }^{14}$ or by limiting observations to a chamber surface $^{15}$. In addition, our findings indicate that many standard 2D chemotaxis assays may be confounded by surface effects in their ability to quantify chemotactic performance, demonstrating the value of 3D tracking.

\section{Multiscale assay captures $C$. crescentus chemotaxis and reveals phenotypically distinct swarmer cell populations}

To demonstrate the power of our approach for revealing novel chemotactic behaviors, we turn to the fresh water bacterium $C$. crescentus whose weak, cell cycle-dependent chemotaxis response ${ }^{16,17}$ has been challenging to capture. In C. crescentus's life cycle, cell division occurs in stalked sessile cells and produces swarmer cells whose "run-reverse-flick" motility ${ }^{9,18}$ is driven by a single polar flagellum. Either direction of flagellar rotation results in locomotion, with the 
flagellum either pushing the cell body forward or pulling it backward. Reversals (turns by $\sim 180^{\circ}$ ) occur with the transition from pushing to pulling, whereas the opposite transition is accompanied by a so-called flick, an approximately right-angle turn (Fig. 2a).

We recorded 79,244 individual 3D bulk trajectories of motile $C$. crescentus cells navigating a $1 \mathrm{mM} / \mathrm{mm}$ xylose gradient (Methods, Supplementary Table 2). Strikingly, 54\% of the more than $123,000 \mathrm{~s}$ of total trajectory time we obtained consists of straight trajectories with no turns and no discernible chemotactic drift up the gradient. A statistical analysis of individual turn event frequencies supports the notion that these "smooth swimmers" form a phenotypically distinct group (Supplementary Discussion). In C. crescentus's life cycle, the swarmer-to-sessile cell transition is accompanied by intracellular biochemical changes that may favor smooth swimming, including the degradation of chemoreceptors ${ }^{17}$ and a rise in c-di-GMP ${ }^{19}$ (Supplementary Discussion). While we do not know the origin of the smooth-swimming population, one possibility is that it represents the early stages of the swarmer-to-sessile cell transition. Smooth swimming is likely to accelerate the rate of surface encounters and may be an integral part of $C$. crescentus's strategy for completing the swarmer-to-sessile cell transition ${ }^{19}$.

The chemotactic drift speed of the remaining, turning population amounts to only $0.26 \pm$ $0.12 \mu \mathrm{m} / \mathrm{s}$ (mean \pm SEM), corresponding to less than $0.5 \%$ of their average swimming speed of $56 \mu \mathrm{m} / \mathrm{s}$. To our knowledge, this is the first measurement of a chemotactic drift speed in C. crescentus, demonstrating the sensitivity enabled by the high throughput of our technique. The speed of backward runs, with the flagellum pulling the cell, is $2.5 \pm 0.2 \%$ higher than that of forward ones (Supplementary Fig. 2e). Forward and backward run duration distributions are approximated well by inverse Gaussian distributions (Supplementary Fig. 2f), consistent with previous reports ${ }^{15,20}$.

\section{C. crescentus performs chemotaxis at near-constant bias}

$C$. crescentus chemotaxis has been assumed to follow the E. coli paradigm, where the cytoplasmic concentration of phosphorylated CheY ([CheY-P]), the chemotaxis response regulator, modulates the fraction of the time that the flagella rotate clockwise, the so-called clockwise bias ${ }^{19,20}$. In E. coli, counterclockwise (CCW) rotation supports locomotion ("runs"), whereas clockwise (CW) rotation induces reorientation events ("tumbles") (Fig. 2b). Chemotaxis is achieved by dynamic modification of the bias so as to increase the duration of runs aligned with the gradient direction. Early studies hypothesized that in $C$. crescentus, forward runs driven by CW rotation correspond to $E$. coli runs, while backward runs driven by $C C W$ rotation are equivalent to $E$. coli tumbles because of their shorter duration ${ }^{21,22}$. Given similar forward and backward swimming speeds, a bias towards $\mathrm{CW}$ rotation would then yield net displacements in the forward swimming direction. In line with this hypothesis, a recent study of 2D surface swimming behavior in oxygen gradients found that forward, but not backward runs, are extended when directed up the gradient compared to down ${ }^{15}$.

39 In contrast with this hypothesis, we find that both forward and backward run segments are 40 extended when ascending, versus descending, a chemoattractant gradient (Fig. 2c, 41 Supplementary Fig. 2f). In fact, our data support a constant motor bias, that is, a constant ratio 42 of forward vs backward swimming interval durations (Fig. 2f) and thus indicate a radical break 43 with the E. coli paradigm of motor bias-driven chemotaxis in $C$. crescentus. We propose that, in 44 C. crescentus, [CheY-P] lowers the energy barrier between the two rotation states, but leaves the 
states' relative energy levels unchanged, thus modulating switching rates without affecting the motor bias ${ }^{10}$ (Fig. 2d, Supplementary Discussion). This interpretation is also consistent with the puzzling previous observation that, in sharp contrast to $E$. coli ${ }^{11}, C$. crescentus shows hardly any variability in motor bias between individuals ${ }^{20}$, suggesting that its motor bias might be unaffected by cytoplasmic fluctuations in [CheY-P].

\section{D tracking overcomes limitations of 2D chemotaxis assays}

The apparent conflict with previous findings ${ }^{15}$ likely results from a technical artefact imposed by the constraints of 2D tracking: to increase the typical time a bacterium spends in the focal plane, 2D bacterial tracking had been performed at the sample chamber surface which hydrodynamically attracts the bacteria ${ }^{23}$. The surface-induced trajectory curvature is more pronounced in the backward than in the forward runs ${ }^{15,24}$ (Supplementary Fig. 2g), thus likely diminishing the chemotactic response more strongly for backward than for forward runs. Placing the focal plane in the bulk can prevent such surface effects in 2D tracking but incurs severely shortened trajectories that are less likely to fully capture runs whose orientation can be assigned based on their bordering turning events. We estimate that, under typical 2D tracking conditions, approximately 30 - 40 times as much data would need to be obtained to detect the dependence of run duration on orientation relative to the gradient at similar fidelity (Supplementary Discussion, Supplementary Figure 3). This example, together with the finding of a decreased drift velocity for $E$. coli close to surfaces, highlights the crucial significance of full 3D behavioral information when assessing chemotactic mechanisms: 3D tracking enables the acquisition of long trajectories without a need for bacterial confinement as well as accurate turning angle measurements for determining bacterial orientation even for short trajectories with few turning events.

\section{Beyond the $E$. coli paradigm}

The mechanism we unveil for the alpha proteobacterium $C$. crescentus aligns with recent findings for the singly flagellated gamma proteobacteria Vibrio alginolyticus ${ }^{9}$ and Pseudomonas aeruginos $a^{10}$. These species also extend both forward and backward swimming intervals during chemotaxis, suggesting that this mechanism may be much more common than previously assumed. To our knowledge, no species with polar flagella has conclusively been shown to follow the $E$. coli scheme, raising the intriguing possibility that the influential $E$. coli paradigm may reflect a special case limited to species that share its flagellation pattern. We note that, despite the stark contrast in motor switching schemes, the resulting swimming pattern suggests a unified behavioral paradigm: any run in a favorable direction is extended.

In summary, our multiscale technique offers unprecedented, simultaneous access to individual and population-level 3D motility behavior and is poised to offer unique insights into novel chemotactic mechanisms as well as into the effects of phenotypic heterogeneity on populationlevel motility behaviors. In contrast to many flow-based chemotaxis assays ${ }^{25}$ that are limited to liquid environments, our assay is compatible with environments such as hydrogels that more closely mimic the complexities of many natural habitats, and thus paves the way for studies of chemotactic mechanisms in ecologically relevant settings. 

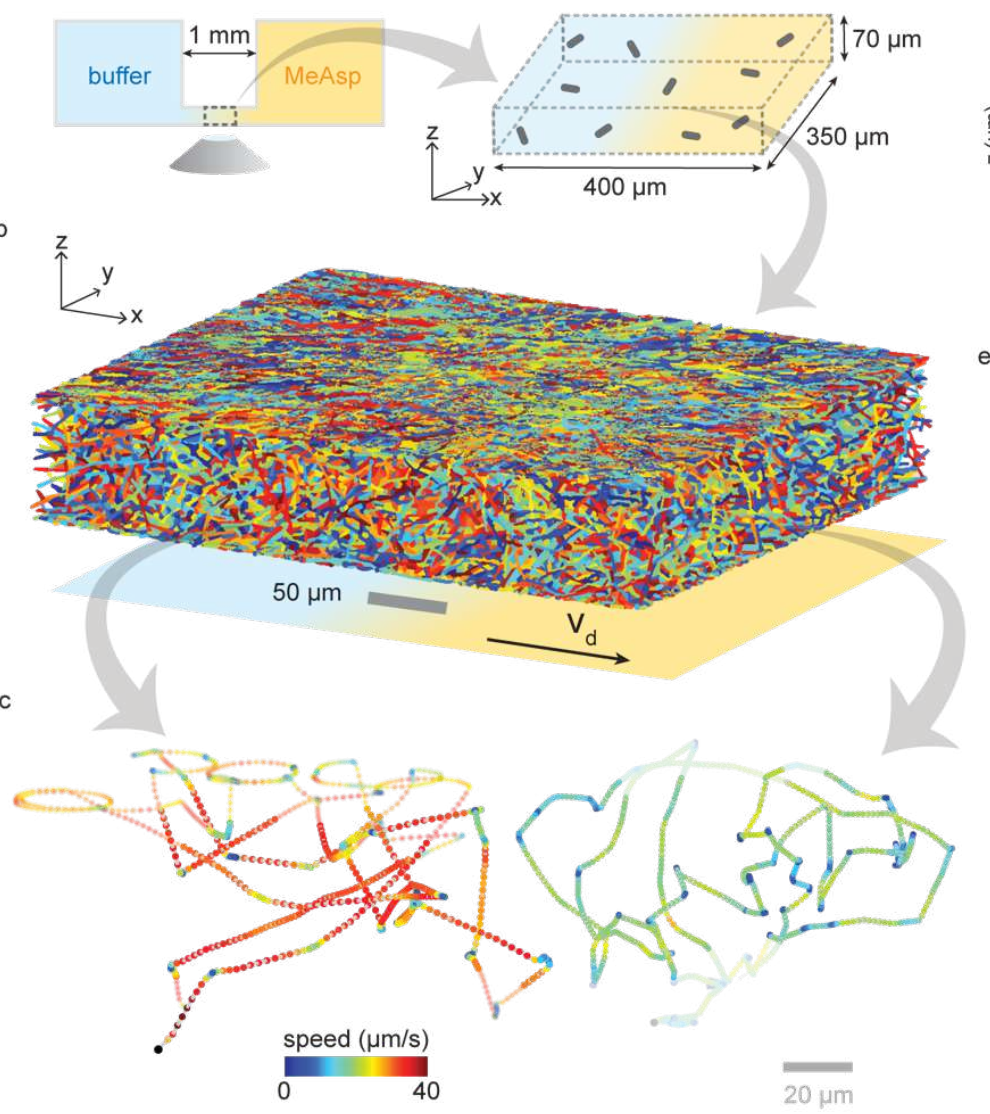
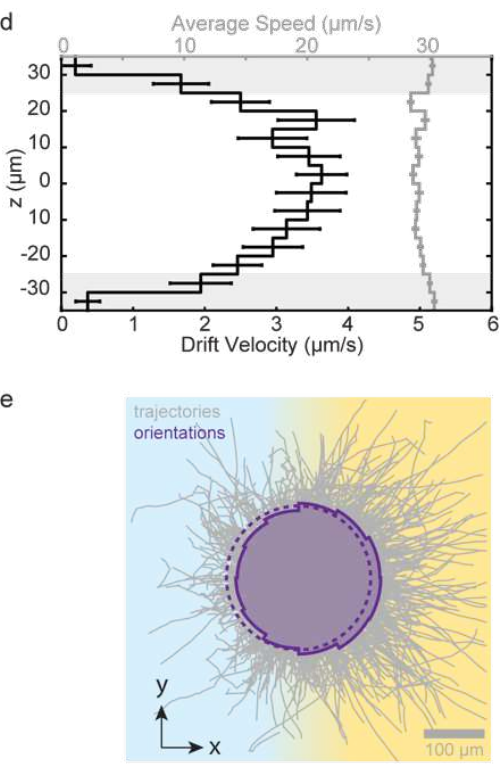

Fig. 1: Schematic of multiscale chemotaxis assay and its application to E. coli strain AW405. a) A quasi-static linear chemical gradient is established between two reservoirs containing a uniform concentration of bacteria. Bacteria are observed in the central portion of the linear gradient. b) 5,045 individual trajectories with a minimum duration of 5 frames and containing 37,080 $\mathrm{s}$ of total trajectory time, obtained in 9 min of recording at $15 \mathrm{~Hz}$ in a typical experiment. c) Two example trajectories (durations $63 \mathrm{~s}$ and $65 \mathrm{~s}$ ) showing run-tumble motility in bulk solution and circular segments near the chamber surface (within $10 \mu \mathrm{m}$ distance, faded). d) Drift velocity (black, defined as the average speed along the gradient direction, $x$ ) and average swimming speed (grey) as a function of height, $z$. Only bulk trajectories (defined as trajectory segments with a distance of more than $10 \mu \mathrm{m}$ to the surface) are retained for further analysis. Error bars reflect standard errors of the mean. e) Bulk trajectories with aligned origins (grey) and polar probability

12 distribution of instantaneous swimming directions projected in the $x-y$ plane (purple, solid line). 13 A flat distribution (dashed) is shown for reference. 
a

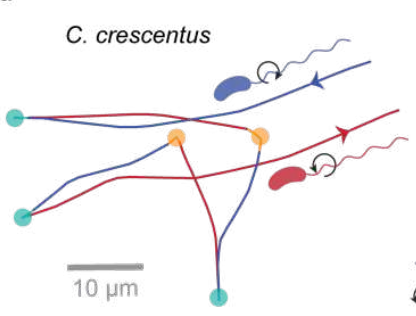

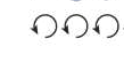

d

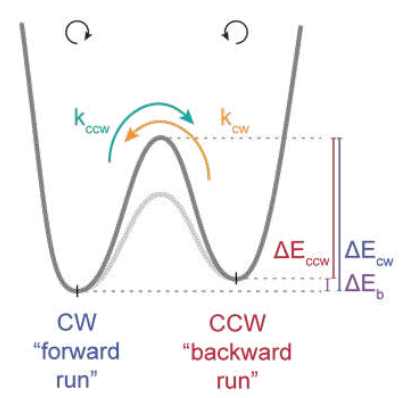

b

e
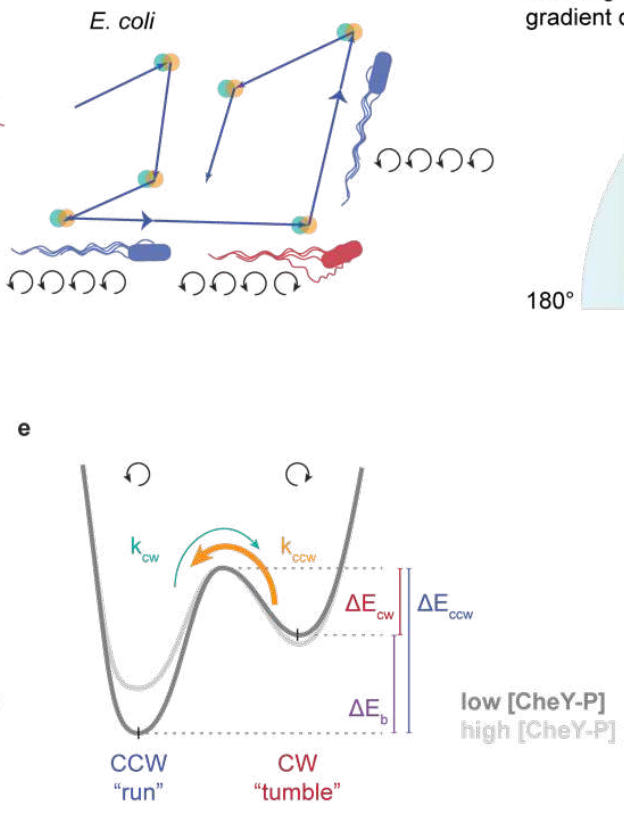

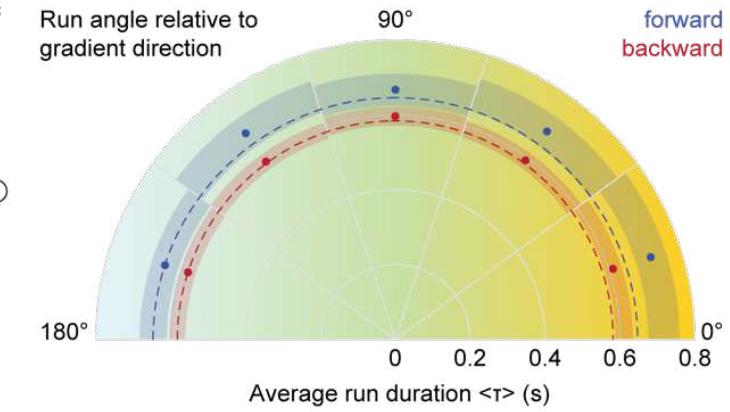

f

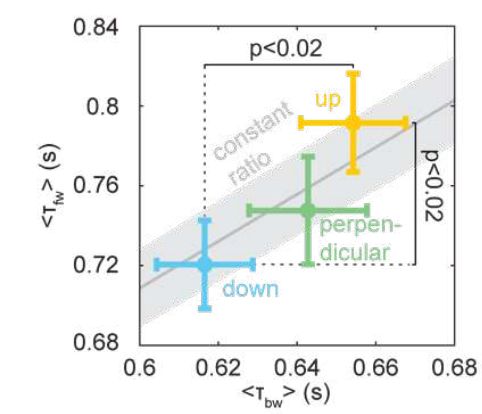

Fig. 2: C. crescentus chemotaxis. a) Example trajectory showing alternating backward (red) and forward (blue) runs, separated by switches in flagellar rotation direction that result in reversals (CW to CCW, teal) or flicks (CCW to CW, orange). b) Schematic of E. coli run-tumble motility. Runs are driven by CCW rotation (blue). Tumbles result from temporary CW rotation (red) of at least one flagellum and thus are bordered by two switches in rotation direction (CCW to CW, teal, CW to CCW, orange). c) Radial plot of average run durations (blue: forward, red: backward runs) as a function of projected angle to the $x$-axis in the $x$ - $y$ plane. The dashed lines indicate the average run durations observed for swimming down the gradient and serve to facilitate comparison. Shading indicates $95 \%$ confidence intervals. d) Schematic of 2-state motor rotation model proposed for $C$. crescentus and e) established for $E$. coli ${ }^{26,27}$. The energy difference $\Delta \mathrm{E}_{\mathrm{b}}$ (purple) between states determines the motor bias, while the energy barrier between states, $\Delta \mathrm{E}_{\mathrm{ccw}}$ and $\Delta \mathrm{E}_{\mathrm{cw}}$, determines the switching rates, $\mathrm{k}_{\mathrm{cw}}$ and $\mathrm{k}_{\mathrm{ccw}}$, respectively (see Supplementary Discussion). f) Average forward versus backward run durations, $\left\langle\tau_{f w}\right\rangle$ versus $\left\langle\tau_{b w}\right\rangle$, up (yellow), down (cyan) or perpendicular to (green) the gradient (defined by $36^{\circ}$ cones around positive $x$-axis, negative $x$ axis, or $y$-axis, respectively). The solid line reflects a best-fit constant CW bias of $0.54 \pm 0.01$, with the standard error (Methods) shown as grey shading. P-values are shown for one-sided t-tests between durations up and down the gradient. Error bars reflect standard error of the mean. 
Methods

Microfluidic device and gradient stability. Quasi-static chemical gradients are created in a commercially available microfluidic device (IBIDI $\mu$-slide Chemotaxis) featuring an approximately $70 \mu \mathrm{m}$ high, $1 \mathrm{~mm}$ long, and $2 \mathrm{~mm}$ wide channel connecting two $65 \mu \mathrm{l}$ reservoirs. Gradient establishment and stability over time were characterized for fluorescein gradients $(10 \mu \mathrm{M} / \mathrm{mm}$ in MotM, see Supplementary Table 1 for media compositions) using a confocal microscope (Zeiss AXIO Imager.Z2). The $488 \mathrm{~nm}$ line was focused through a 20x water immersion objective and fluorescence emission collected in the $500-585 \mathrm{~nm}$ window with a pinhole adjusted to $30 \mu \mathrm{m}$ diameter. A $4.5 \mathrm{~h}$ time series of line scans in the gradient direction across the center of the device was acquired after closing the device (Supplementary Fig. 1b). A large-scale $4 \times 4 \mathrm{~mm}$ view of the device was obtained by tiling 2D scans (Supplementary Fig. 1a) acquired $5 \mathrm{~h}$ after closing the device. The gradient is established within minutes of filling the device and shows a deviation of less than $4 \%$ from the final plateau value after 30 minutes (Supplementary Fig. 1c). No detectable variation in relative gradient magnitude is observed in the time range from $40 \mathrm{~min}$ to $4.5 \mathrm{~h}$ after filling the device (Supplementary Fig. 1c).

\section{E. coli experiments}

Bacterial culturing. Overnight cultures were inoculated from a frozen glycerol stock of $E$. coli AW405 (a kind gift of Howard Berg) in $2 \mathrm{ml}$ TB and grown to saturation at $30^{\circ} \mathrm{C}, 250 \mathrm{rpm}$. Day cultures were inoculated with the overnight cultures at 1:200 dilution in $10 \mathrm{ml} \mathrm{TB}$ and grown at $33.5^{\circ} \mathrm{C}, 250 \mathrm{rpm}$, until they reached an optical density (OD) between 0.3 and 0.35 at $600 \mathrm{~nm}$. Volumes of $1 \mathrm{ml}$ of bacterial culture were washed by three rounds of centrifugation in $1.5 \mathrm{ml}$ microcentrifuge tubes ( $6 \mathrm{~min}$ at 2,000 rcf), each followed by gentle resuspension in $1 \mathrm{ml}$ of motility medium MotM. They were diluted to a target OD of 0.003 (for acquisitions in a gradient) or 0.005 (no gradient) in MotM supplemented with $0.002 \%$ Tween 20 , with or without chemoattractant, for injection into the chemotaxis device.

Sample preparation. The device's reservoirs were filled with the two bacterial solutions (with or without chemoattractant) following a modified version of the manufacturer's "Fast Method" protocol. First, the entire device was overfilled with buffer free of chemoattractant or bacteria through the filling ports, and then the central channel's ports were closed with plugs. $65 \mu$ l was removed from one reservoir, replaced by $65 \mu \mathrm{l}$ of chemoattractant-free bacterial solution, and then this reservoir's ports were closed. Finally, all liquid was removed from the other reservoir and replaced with bacterial solution containing chemoattractant. Key to reproducible gradients is to not overfill this reservoir to avoid liquid flow in the central channel when the last two ports are closed. A uniform bacterial density across the device ensures that any population drift observed is not the result of a diffusive flux, but likely indicates chemotaxis. For control measurements, neither bacterial solution contained chemoattractant. At the bacterial densities used (OD of 0.005 or less), oxygen depletion is unlikely, and we do not observe a change in $E$. coli swimming speed in the reservoirs over the course of $1 \mathrm{~h}$ (Supplementary Fig. 1d). 
Data acquisition. Phase contrast microscopy recordings were obtained at room temperature $\left(\sim 22^{\circ} \mathrm{C}\right)$ on a Nikon Ti-E inverted microscope using an sCMOS camera (PCO Edge 4.2) and a 40x objective lens (Nikon CFI SPlan Fluor ELWD 40x ADM Ph2, correction collar set to $1.2 \mathrm{~mm}$ to induce spherical aberrations ${ }^{12}$ ) focused at the center of the channel in all three dimensions. 3D bacterial trajectories were extracted ${ }^{12}$ for a field of view of $\sim 350 \mu \mathrm{m} \times 300 \mu \mathrm{m}$ laterally $(x, y)$ and over the entire depth $(z)$ of the channel for typically several dozen individuals at a time. For $E$. coli, recordings were obtained starting from 50 minutes after filling the device. Three 3-min long recordings were obtained at $15 \mathrm{fps}$. Three replicates can be performed in parallel in half a day. In-device conditions. We confirmed that the E. coli motile population we tracked in the central channel was representative of the whole population by also acquiring trajectories in one chamber during an experiment. The average speed of the motile population, defined as individuals having a mean swimming speed larger than $10 \mu \mathrm{m} / \mathrm{s}$, in the reservoirs was stable in time and similar to that observed in the central channel (Supplementary Fig. 1d).

Data analysis. 3D Trajectories were extracted from phase contrast recordings using a highthroughput 3D tracking method based on image similarity between bacteria and a reference library ${ }^{12}$. Trajectories shorter than 5 frames were discarded. Positions were smoothed using $2^{\text {nd }}$ order ADMM-based trend-filtering ${ }^{28}$ with regularization parameter $\lambda=1$, and speeds computed as forward differences in positions divided by the time interval between frames. All trajectories with an average speed below a threshold were considered non-motile and discarded. The threshold was set at $15 \mu \mathrm{m} / \mathrm{s}$ unless noted otherwise. The $z$ position of the top and bottom of the chamber were identified by visual inspection of trajectory data. All trajectory segments within $10 \mu \mathrm{m}$ of the top or bottom of the central channel were removed to avoid surface interaction effects, retaining $35 \%$ of total trajectory time. Fig. $1 \mathrm{~b}$ shows data for one replicate. For Fig. $1 \mathrm{~d}$ and e, we combine data from three biological repeats each yielding three recordings. The profile of the drift velocity as function of $z$ position is similar in each replicate (Supplementary Fig. 1f). The drift velocity is the average of the $x$ component of all 3D speed vectors from all bacteria. Across 3 biological replicates performed in parallel, we obtain a drift velocity of $2.7 \pm 0.3 \mu \mathrm{m} / \mathrm{s}$ (mean \pm SD across the replicates) for $E$. coli in a $10 \mu \mathrm{M} / \mathrm{mm}$ MeAsp gradient, and observe no chemotactic drift along either the $y$ or $z$ axis $(0.1 \pm 0.3 \mu \mathrm{m} / \mathrm{s}$ and $0.09 \pm 0.1 \mu \mathrm{m} / \mathrm{s}$, respectively). Biological replicates performed on other days yielded drift velocities of $2.7 \mu \mathrm{m} / \mathrm{s}$ and $2.5 \mu \mathrm{m} / \mathrm{s}$. A control chamber without a gradient showed no drift either $(0.34 \pm 0.8 \mu \mathrm{m} / \mathrm{s}$ along $x)$. For data obtained from a single experiment (Supplementary Fig. 1e), we estimate the noise on the drift measurement by a jackknife resampling procedure consisting of dividing the data into subsets of 150 trajectories and computing the standard error of the mean drift obtained for different subsets. For drift as a function of $z$, trajectories are first sliced into segments by $z$ bin, and jackknifing is performed for each $z$ bin.

\section{C. crescentus experiments}

Bacterial culturing and sample preparation. Overnight cultures were inoculated from individual C. crescentus (CB15, ATCC 19089) colonies, grown on 1.5\% agar PYE plates streaked from glycerol stock, and grown to saturation in $2 \mathrm{ml} \mathrm{PYE} \mathrm{at} 30^{\circ} \mathrm{C}, 200 \mathrm{rpm}$. Day cultures were inoculated at a dilution of $1: 20\left(\mathrm{v} / \mathrm{v}\right.$ ) in $\mathrm{M}_{2} \mathrm{G}^{29}$ or PYE and grown to an OD600 of at least 0.3 (a few hours for PYE, 
around 12 hours for M2G). Because the cell-cycle dependent motility of $C$. crescentus is quickly lost, we opted to grow the cells directly inside the device and let them produce swarmer cells while the gradient is being established. To this end, the day culture was again diluted 1:1 in fresh medium and injected into both reservoirs of the device which was then incubated at room temperature. When the chamber walls were colonized by a sufficient density of stalked cells as determined by visual inspection under the microscope (a few hours for PYE, 2 days for M2G), the device was rinsed several times with fresh $\mathrm{M} 2 \mathrm{G}$, until no swimming bacteria were observed. Then fresh $\mathrm{M} 2 \mathrm{G}$ and $1 \mathrm{mM}$ xylose/M2G, respectively, were injected into the reservoirs to create a $1 \mathrm{mM} / \mathrm{mm}$ xylose gradient in the central channel. Xylose is a known chemoattractant for $C$. crescentus $^{21,30}$.

Data acquisition. Because of the cell cycle-dependent chemotaxis ${ }^{16}$ of $C$. crescentus, we favored acquiring data as early as possible over waiting for perfect gradient stability. Recordings spanning 2.5 minutes at $30 \mathrm{fps}$ were acquired from 20 to 55 minutes after closing the device, in five biologically independent experiments, totaling a cumulated acquisition time of 75 minutes.

Data analysis. Trajectories were obtained and smoothed as for E. coli, except for the ADMM regularization parameter being set to $\lambda=0.3$. To account for the layer of attached cells lining the surface, only segments with a distance of more than $13 \mu \mathrm{m}$ from the top or bottom chamber surface were retained to avoid surface interaction effects. Supplementary Table 2 details statistical characteristics of the subsets of data used for analysis. Standard errors on drift velocities are obtained by jackknifing as described for $E$. coli.

Run-reverse-flick analysis. The turning event detection is based on the local rate of angular change, computed from the dot product between the sums of the two consecutive velocity vectors preceding and subsequent to a time point. The threshold for a turn to begin is an $\alpha$-fold rate relative to the median rate of angular change rate of the run segments, as determined in three iterations of the procedure. We determined by visual inspection of trajectories that a factor $\alpha=6$ gave satisfactory results. A new run begins with at least two time points (at least $0.066 \mathrm{~s}$ ) under this threshold. Backward (CCW rotation) and forward (CW rotation) runs were identified as runs with a turn under $130^{\circ}$, respectively at the end or at the beginning the run, and a turn above $150^{\circ}$ at the other end of the run. A total of 5,342 backward and 2,898 forward runs were identified within a subpopulation of 6,230 trajectories.

Run duration analysis. Runs are considered to be going up or down the gradient if they fall within a $36^{\circ}$ cone around the positive, respectively negative, $x$ axis. The value of $36^{\circ}$ was chosen so as to balance a trade-off between maximizing the number of contributing runs with maximizing their alignment with the gradient direction. The conclusions are not sensitive to the exact value chosen. The fact that runs going up the gradient are longer than those going down the gradient confirms that the observed drift, though small, is indeed caused by chemotaxis. We determine maximum-likelihood inverse Gaussian distributions from the run duration data:

$$
f=\sqrt{\frac{\lambda}{2 \pi \tau^{3}}} \exp \left(-\frac{\lambda(\tau-\mu)^{2}}{2 \mu^{2} \tau}\right)
$$

where the run duration $\tau$, the mean, $\mu$, and the shape parameters, $\lambda$, are strictly positive. We obtain the following parameters: $\mu=0.79 \mathrm{~s}, \lambda=1.18 \mathrm{~s}$, and $\mu=0.72 \mathrm{~s}, \lambda=1.21 \mathrm{~s}$, for forward runs going up and down the gradient, respectively; and $\mu=0.65 \mathrm{~s}, \lambda=1.85 \mathrm{~s}$, and $\mu=0.62 \mathrm{~s}, \lambda=$

$421.78 \mathrm{~s}$, for backward runs going up or down the gradient, respectively. 
1 Motor bias analysis. To determine a best-fit motor bias for Fig. 2c, an orthonormal linear fit 2 constrained to a zero intercept is applied to the average run durations up, down, and 3 perpendicular to the gradient. The error on the slope is estimated as the standard deviation of 4 slopes obtained by fitting data generated in a Monte Carlo procedure, consisting of drawing data 5 points randomly from Gaussian distributions centered about the actual data points and with a 6 standard deviation matching the data points' standard error. The $\mathrm{CW}$ bias, $b_{\mathrm{CW}}$, can be obtained 7 from the slope, $s$, as $b_{\mathrm{cW}}=s /(1+s)$. We obtain $s=1.18 \pm 0.03$, corresponding to $b_{\mathrm{cw}}=0.54 \pm 0.01$. 8 Run speed analysis. To determine the ratio of forward to backward swimming speed for 9 Supplementary Fig. 2e, average speeds were computed across all forward and backward runs, 10 respectively, for each trajectory in subset 8 (see Supplementary Table 2). An orthonormal linear 11 fit constrained to zero intercept yields a slope of $1.025 \pm 0.002$ (SE).

12

13

14 


\begin{tabular}{|c|c|c|}
\hline \multirow{2}{*}{$\begin{array}{l}1 \\
2\end{array}$} & \multicolumn{2}{|c|}{ References } \\
\hline & & \\
\hline 4 & 1 & Matilla, M. A. \& Krell, T. The effect of bacterial chemotaxis on host infection and \\
\hline 5 & & pathogenicity. FEMS Microbiology Reviews 42, 202, doi:10.1093/femsre/fux052 (2018). \\
\hline 6 & 2 & Raina, J.-B., Fernandez, V., Lambert, B., Stocker, R. \& Seymour, J. R. The role of microbial \\
\hline 7 & & motility and chemotaxis in symbiosis. Nature Reviews Microbiology 292, 1096, \\
\hline 8 & & doi:10.1038/s41579-019-0182-9 (2019). \\
\hline 9 & 3 & Seymour, J. R., Amin, S. A., Raina, J.-B. \& Stocker, R. Zooming in on the phycosphere: the \\
\hline 10 & & ecological interface for phytoplankton-bacteria relationships. Nature microbiology $\mathbf{2}$ \\
\hline 11 & & 17065, doi:10.1038/nmicrobiol.2017.65 (2017). \\
\hline 12 & 4 & Adler, J. A Method for Measuring Chemotaxis and Use of the Method to Determine \\
\hline 13 & & Optimum Conditions for Chemotaxis by Escherichia coli. Journal of general microbiology \\
\hline 14 & & 74, 77-91, doi:10.1099/00221287-74-1-77 (1973). \\
\hline 15 & 5 & Colin, R., Zhang, R. \& Wilson, L. G. Fast, high-throughput measurement of collective \\
\hline 16 & & behaviour in a bacterial population. Journal of The Royal Society Interface 11, 20140486- \\
\hline 17 & & 20140486, doi:10.1098/rsif.2014.0486 (2014). \\
\hline 18 & 6 & Berg, H. C. \& Brown, D. A. Chemotaxis in Escherichia coli analysed by Three-dimensional \\
\hline 19 & & Tracking. Nature 239, 500-504, doi:10.1038/239500a0 (1972). \\
\hline 20 & 7 & Larsen, S. H., Reader, R. W., Kort, E. N., Tso, W.-w. \& Adler, J. Change in direction of \\
\hline 21 & & flagellar rotation is the basis of the chemotactic response in Escherichia coli. Nature 249, \\
\hline 22 & & 74-77, doi:10.1038/249074a0 (1974). \\
\hline 23 & 8 & Webre, D. J., Wolanin, P. M. \& Stock, J. B. Bacterial chemotaxis. Current Biology 13, R47- \\
\hline 24 & & 49 (2003). \\
\hline 25 & 9 & Xie, L., Altindal, T., Chattopadhyay, S. \& Wu, X.-I. Bacterial flagellum as a propeller and \\
\hline 26 & & as a rudder for efficient chemotaxis. Proceedings of the National Academy of Sciences \\
\hline 27 & & 108, 2246-2251, doi:10.1073/pnas.1011953108 (2011). \\
\hline 28 & 10 & Cai, Q., Li, Z., Ouyang, Q., Luo, C. \& Gordon, V. D. Singly Flagellated Pseudomonas \\
\hline 29 & & aeruginosa Chemotaxes Efficiently by Unbiased Motor Regulation. mBio 7, e00013, \\
\hline 30 & & doi:10.1128/mBio.00013-16 (2016). \\
\hline 31 & 11 & Spudich, J. L. \& Koshland, D. E. Non-genetic individuality: chance in the single cell. \\
\hline 32 & & Nature 262, 467-471 (1976). \\
\hline 33 & 12 & Taute, K. M., Gude, S., Tans, S. J. \& Shimizu, T. S. High-throughput 3D tracking of \\
\hline 34 & & bacteria on a standard phase contrast microscope. Nature Communications 6, 1-9, \\
\hline 35 & & \\
\hline 36 & 13 & Lauga, E., DiLuzio, W. R., Whitesides, G. M. \& Stone, H. a. Swimming in Circles: Motion of \\
\hline 37 & & Bacteria near Solid Boundaries. Biophysical Journal 90, 400-412, \\
\hline 38 & & doi:10.1529/biophysj.105.069401 (2006). \\
\hline 39 & 14 & Waite, A. J. et al. Non - genetic diversity modulates population performance. Molecular \\
\hline 40 & & Systems Biology 12, 895-814, doi:10.15252/msb.20167044 (2016). \\
\hline 41 & 15 & Morse, M., Colin, R., Wilson, L. G. \& Tang, J. X. The Aerotactic Response of Caulobacter \\
\hline 42 & & crescentus. Biophysical Journal 110, 2076-2084, doi:10.1016/j.bpj.2016.03.028 (2016). \\
\hline
\end{tabular}




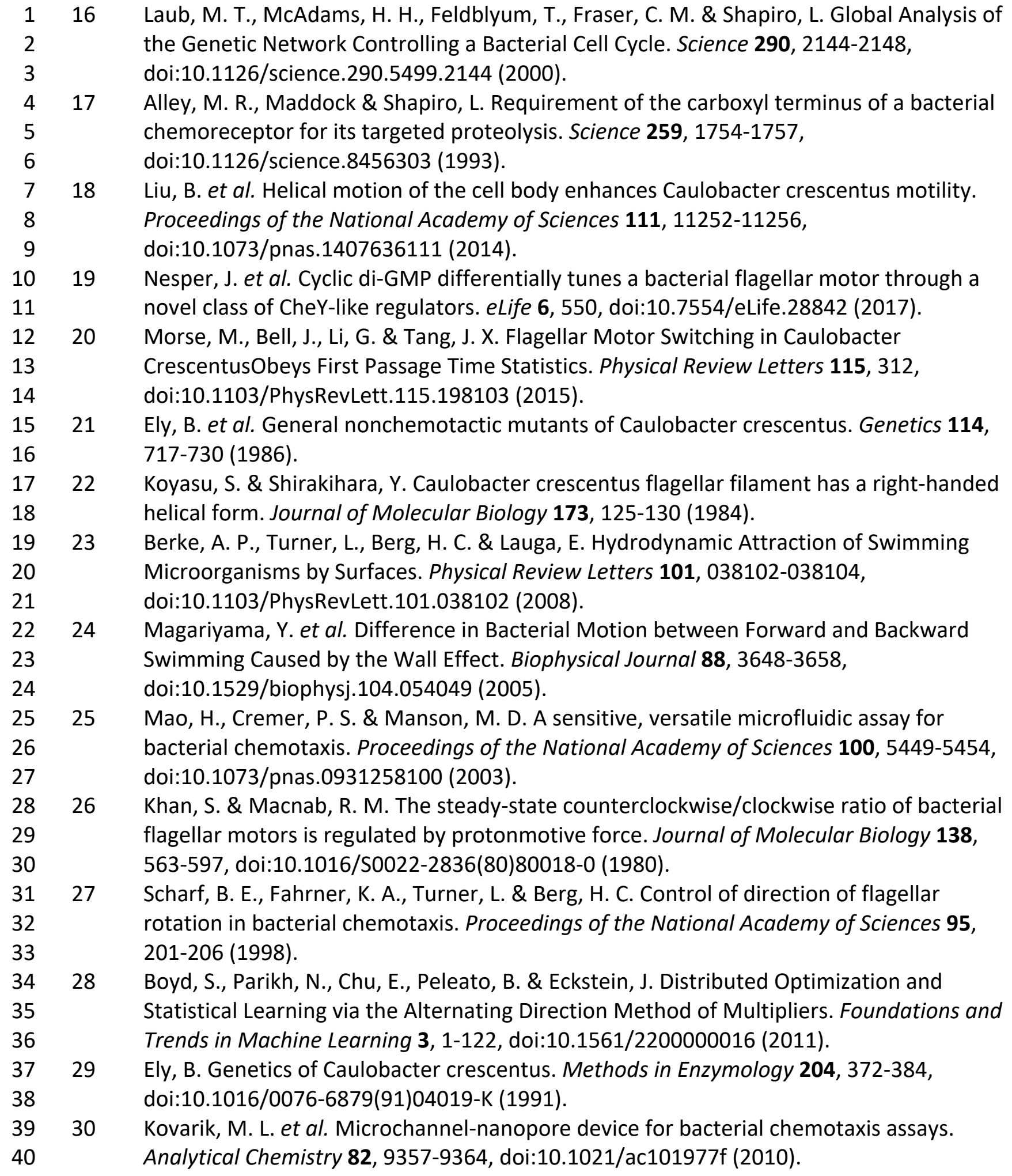

41

42

43 


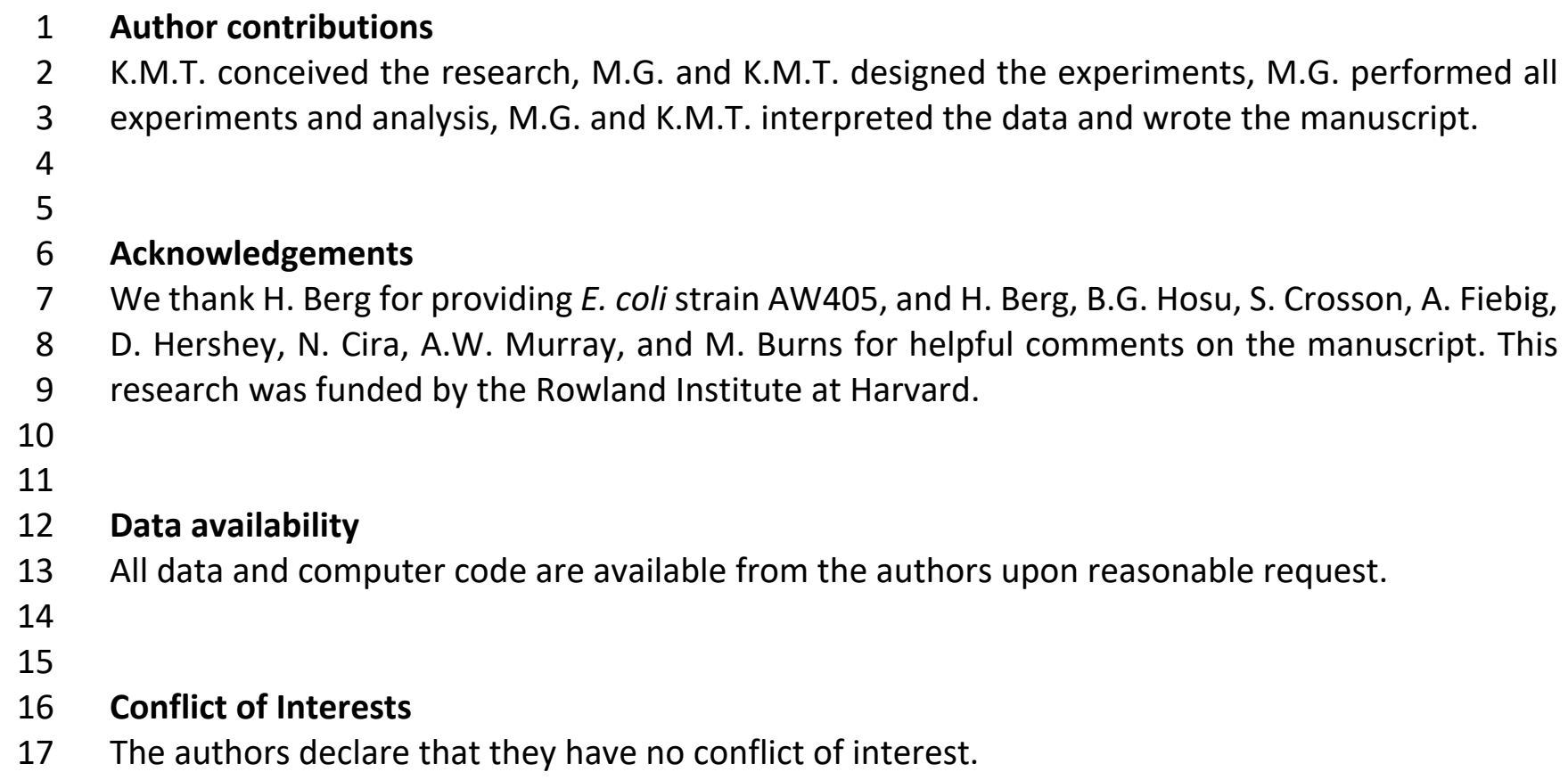

\section{Conflict of Interests}

17 The authors declare that they have no conflict of interest. 


\section{Figures}

a

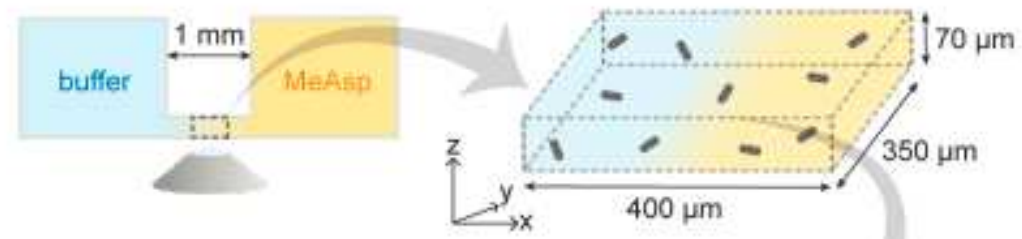

b

$\sum^{\mathrm{z}} \mathrm{z}$

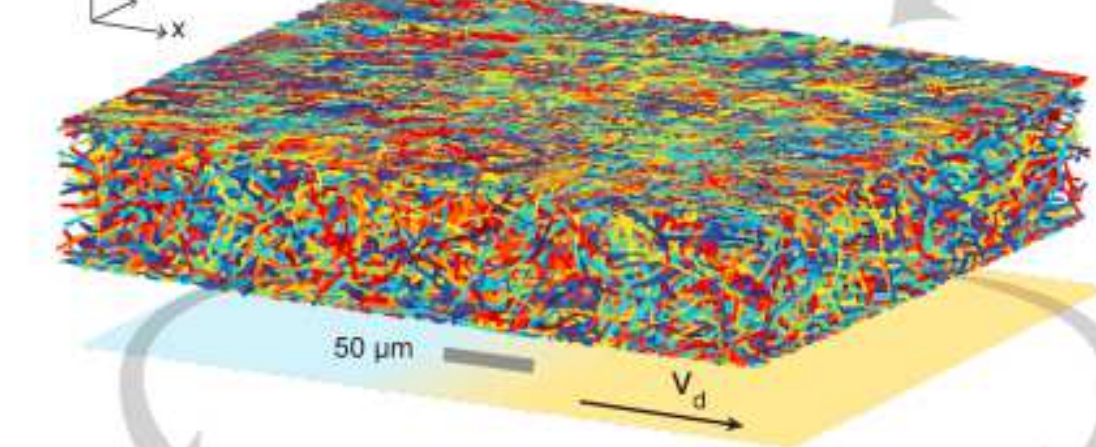

c

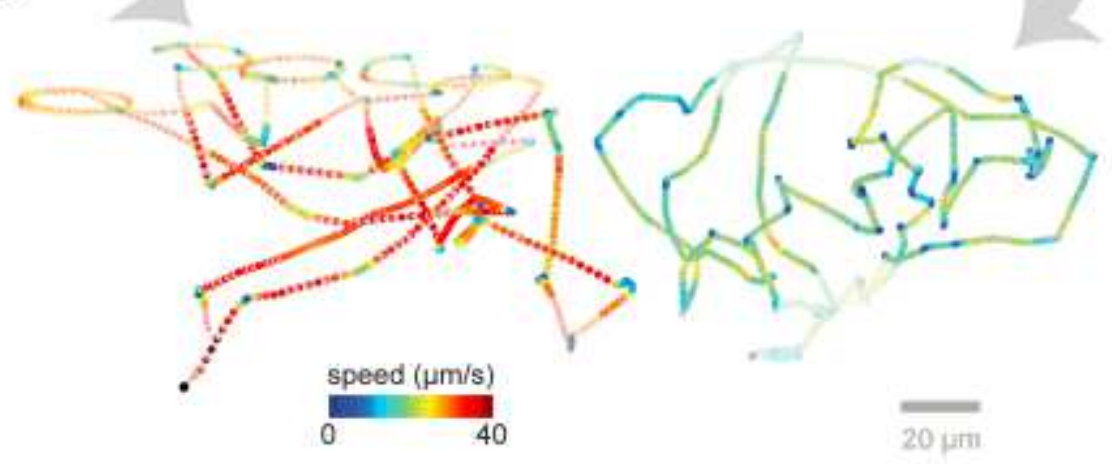

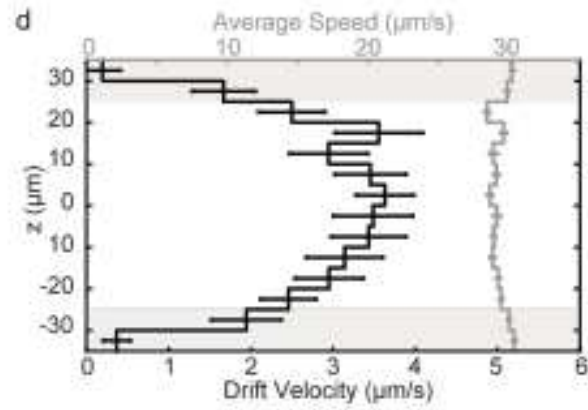

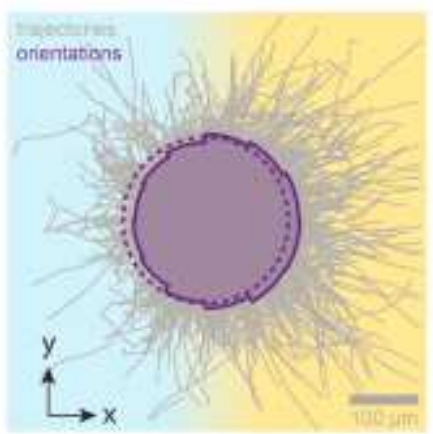

\section{Figure 1}

Schematic of multiscale chemotaxis assay and its application to E. coli 1 strain AW405. a) A quasi-static linear chemical gradient is established between two reservoirs containing a uniform concentration of bacteria. Bacteria are observed in the central portion of the linear gradient. b) 5,045 individual trajectories with a minimum duration of 5 frames and containing 37,080 s of total trajectory time, obtained in 9 min of recording at $15 \mathrm{~Hz}$ in a typical experiment. c) Two example trajectories (durations $63 \mathrm{~s}$ and $65 \mathrm{~s}$ ) showing run-tumble motility in bulk solution and circular segments near the chamber surface (within 10 $\mu \mathrm{m}$ distance, faded). d) Drift velocity (black, defined as the average speed along the gradient direction, $\mathrm{x}$ ) and average swimming speed (grey) as a function of height, z. Only bulk trajectories (defined as trajectory segments with a distance of more than $10 \mu \mathrm{m}$ to the surface) are retained for further analysis. Error bars reflect standard errors of the mean. e) Bulk trajectories with aligned origins (grey) and polar probability distribution of instantaneous swimming directions projected in the $x-y$ plane (purple, solid line). A flat distribution (dashed) is shown for reference. 


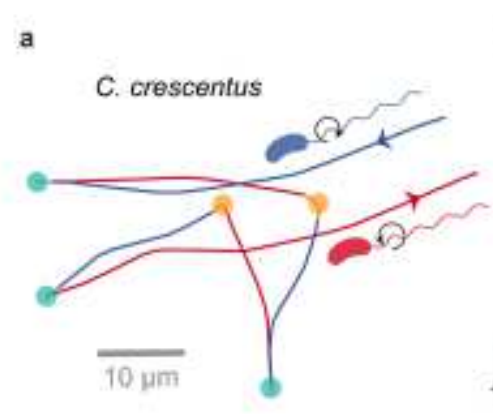

b
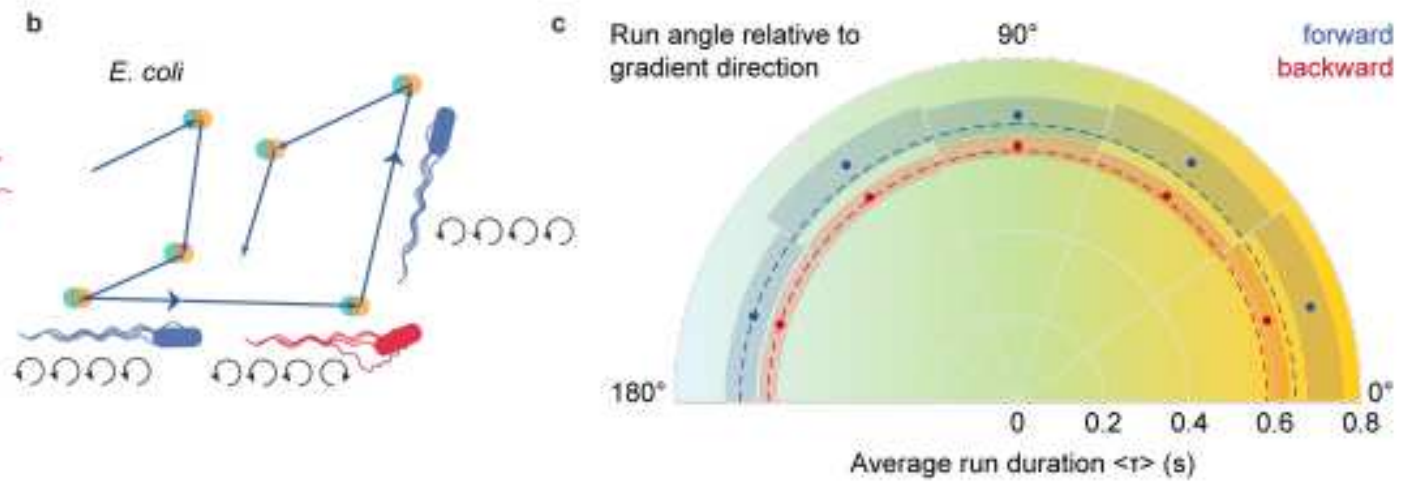

d

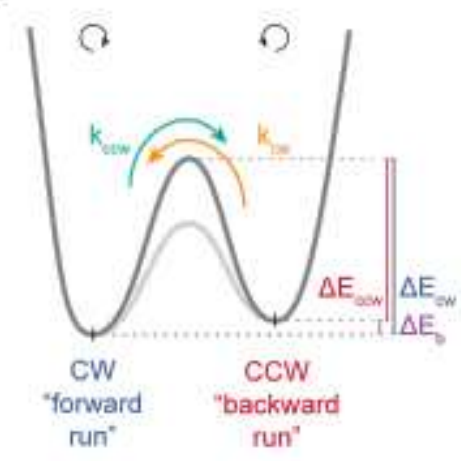

e

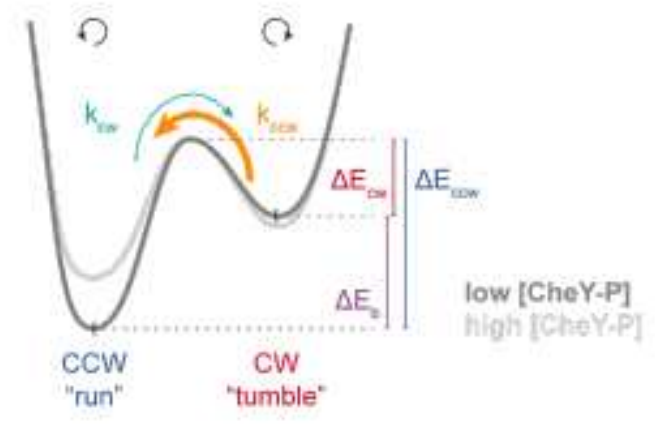

$f$

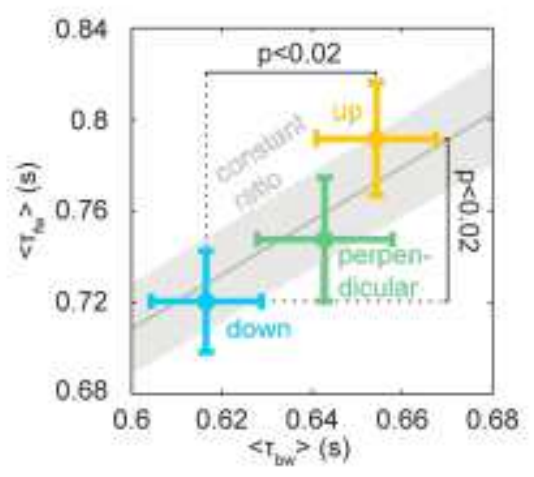

\section{Figure 2}

C. crescentus chemotaxis. a) Example trajectory showing alternating backward (red) and forward (blue) runs, separated by switches in flagellar rotation direction that result in reversals (CW to CCW, teal) or flicks (CCW to CW, orange). b) Schematic of E. coli run-tumble motility. Runs are driven by CCW rotation (blue). Tumbles result from temporary $\mathrm{CW}$ rotation (red) of at least one flagellum and thus are bordered by two switches in rotation direction (CCW to $\mathrm{CW}$, teal, $\mathrm{CW}$ to $\mathrm{CCW}$, orange). c) Radial plot of average run durations (blue: forward, red: backward runs) as a function of projected angle to the $x$-axis in the $x-y$ plane. The dashed lines indicate the average run durations observed for swimming down the gradient and serve to facilitate comparison. Shading indicates $95 \%$ confidence intervals. d) Schematic of 2-state motor rotation model proposed for C. crescentus and e) established for E. coli26,27. The energy difference $\Delta E b$ (purple) between states determines the motor bias, while the energy barrier between states, $\Delta \mathrm{Eccw}$ and $\Delta \mathrm{Ecw}$, determines the switching rates, $\mathrm{kcw}$ and $\mathrm{kccw}$, respectively (see Supplementary Discussion). f) Average forward versus backward run durations, $\langle\tau f w>$ versus $<\tau b w>$, up (yellow), down (cyan) or perpendicular to (green) the gradient (defined by $36^{\circ}$ cones around positive $x$-axis, negative $x$ axis, or $y$-axis, respectively). The solid line reflects a best-fit constant $\mathrm{CW}$ bias of $0.54 \pm 0.01$, with the standard error (Methods) shown as grey shading. P-values are shown for one-sided t-tests between durations up and down the gradient. Error bars reflect standard error of the mean.

\section{Supplementary Files}

This is a list of supplementary files associated with this preprint. Click to download. 
- Supplementarylnformation.pdf 